REPRESENTATION THEORY

An Electronic Journal of the American Mathematical Society

Volume 3, Pages 457-473 (December 3, 1999)

S $1088-4165(99) 00084-9$

\title{
DIFFERENTIAL OPERATORS ON SOME NILPOTENT ORBITS
}

\author{
T. LEVASSEUR AND J. T. STAFFORD
}

\begin{abstract}
In recent work, Astashkevich and Brylinski construct some differential operators of Euler degree -1 (thus, they lower the degree of polynomials by one) on the coordinate ring $\mathcal{O}\left(\mathbb{O}_{\min }(\mathfrak{g})\right)$ of the minimal nilpotent orbit $\mathbb{O}_{\text {min }}(\mathfrak{g})$ for any classical, complex simple Lie algebra $\mathfrak{g}$. They term these operators "exotic" since there is "(apparently) no geometric or algebraic theory that explains them".

In this paper, we provide just such an algebraic theory for $\mathfrak{s l}(n)$ by giving a complete description of the ring of differential operators on $\mathbb{O}_{\min }(\mathfrak{s l}(n))$. The method of proof also works for various related varieties, notably for the Lagrangian submanifolds of the minimal orbit of classical Lie algebras for which Kostant and Brylinski have constructed exotic differential operators.
\end{abstract}

\section{INTRODUCTION}

In [1, Astashkevich and Brylinski construct some differential operators of Euler degree -1 (thus, they lower the degree of polynomials by one) on the coordinate ring $\mathcal{O}\left(\mathbb{O}_{\min }(\mathfrak{g})\right)$ of the minimal nilpotent orbit $\mathbb{O}_{\min }(\mathfrak{g})$ for any classical, complex simple Lie algebra $\mathfrak{g}$. They term these operators "exotic" since there is "(apparently) no geometric or algebraic theory that explains them".

The aim of this paper is to provide just such an algebraic theory for $\mathfrak{s l}(n)$, $n \geq 2$, by giving a complete description of the ring of differential operators $\mathcal{D}(\mathbb{O})$ defined over $\mathbb{O}=\mathbb{O}_{\min }(\mathfrak{s l}(n))$. The method of proof also works for various related varieties, notably for the Lagrangian submanifolds of the minimal orbit of classical Lie algebras, as defined in [6, 7, 9]. As with those papers, our results can be thought of as a particular case of geometric quantization, and for a general discussion of the relationship the reader is referred to [1, 6, 7, 8, 9, 10,

In order illustrate the techniques involved, consider the minimal orbit $\mathbb{O}$ in $\mathfrak{s l}(n)$. Then the closure $\overline{\mathbb{O}}$ of $\mathbb{O}$ is the set of $n \times n$ matrices of trace zero and rank less than or equal to one. As such, it can be identified with the set of closed GL(1)-orbits in

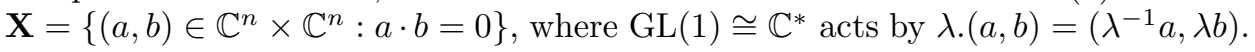
From earlier work of the authors, one may explicitly describe the ring of differential operators $\mathcal{D}(\mathbf{X})$ on $\mathbf{X}$; indeed, $\mathcal{D}(\mathbf{X}) \cong U(\mathfrak{s o}(2 n+2)) / J_{0}$ where, for $n \geq 3, J_{0}$ is the Joseph ideal. On the other hand, restriction of functions induces a map $\rho: \mathcal{D}(\mathbf{X})^{\mathbb{C}^{*}} \rightarrow \mathcal{D}(\mathbb{O})$. Using these observations, it is very easy to write down the exotic elements of $\mathcal{D}(\mathbb{O})$, as we show in Section 1 .

Received by the editors September 7, 1999 and, in revised form, October 13, 1999.

1991 Mathematics Subject Classification. Primary 14L30, 16S32, 17B20, 58F06.

Key words and phrases. Reductive Lie algebras, nilpotent orbits, differential operators, geometric quantization.

The research of both authors was supported in part by NSF grant NSF-G-DMS 9801148. 
However, this technique is still more powerful, since the main result of this paper shows that $\rho$ is surjective for $n \geq 3$ and hence describes all the differential operators on $\mathbb{O}$. Let $\tau: \mathfrak{g l}(1)=\mathbb{C} \rightarrow \mathcal{D}(\mathbf{X}) \cong U(\mathfrak{s o}(2 n+2)) / J_{0}$ denote the differential of the GL(1)-action on $\mathbf{X}$. Then:

Theorem 0.1. Assume that $n \geq 3$. Then, the minimal orbit $\mathbb{O}=\mathbb{O}_{\min }(\mathfrak{s l}(n))$ in $\mathfrak{s l}(n)$ has a ring of differential operators:

$$
\mathcal{D}(\mathbb{O}) \cong \frac{\left(U(\mathfrak{s o}(2 n+2)) / J_{0}\right)^{\mathrm{GL}(1)}}{(\tau(\mathfrak{g l}(1)))} .
$$

For any simple Lie algebra $\mathfrak{g}, \overline{\mathbb{O}}_{\min }(\mathfrak{g}) \backslash \mathbb{O}_{\min }(\mathfrak{g})$ has codimension at least two in the normal affine variety $\overline{\mathbb{O}}_{\min }(\mathfrak{g})$, and so [19, Proposition 2, p. 167] implies that $\mathcal{O}\left(\mathbb{O}_{\min }(\mathfrak{g})\right)=\mathcal{O}\left(\overline{\mathbb{O}}_{\text {min }}(\mathfrak{g})\right)$ and $\mathcal{D}\left(\mathbb{O}_{\min }(\mathfrak{g})\right)=\mathcal{D}\left(\overline{\mathbb{O}}_{\text {min }}(\mathfrak{g})\right)$. Thus, it does not matter whether we work with the orbit or its closure in this, or any related theorem.

When $n=2$, one cannot talk about the Joseph ideal, since $\mathfrak{s o}(6) \cong \mathfrak{s l}(4)$. However, the map $\rho: \mathcal{D}(\mathbf{X})^{\mathbb{C}^{*}} \rightarrow \mathcal{D}(\overline{\mathbb{O}})$ still exists, and so one can still ask whether $\rho$ is surjective. It is not (see Section 3 ).

We are unable to prove an analogue of Theorem 0.1 for the minimal orbit in $\mathfrak{s o}(n)$ since there is not a sufficiently simple description of $\overline{\mathbb{O}}_{\min }(\mathfrak{s o}(n))$ as a categorical quotient. See Section [6] for a more detailed discussion of this and other open questions. As noted in [1], the remaining case of $\mathfrak{s p}(2 n)$ is less interesting since, here, $\mathcal{D}\left(\overline{\mathbb{O}}_{\text {min }}(\mathfrak{s p}(2 n))\right)$ is simply the even degree elements of the Weyl algebra $A_{2 n}(\mathbb{C})$.

In Section 4 we prove an analogue to Theorem 0.1 for the Lagrangian submanifolds $\mathbf{L}=\overline{\mathbb{O}}_{\text {min }}(\mathfrak{g}) \cap \mathfrak{p}$ of classical simple Lie algebras $\mathfrak{g}$ that were considered in $[9]$. These varieties arise as follows: Let $\mathfrak{g}_{\mathbb{R}}$ be a simple, real Lie algebra with a Cartan decomposition $\mathfrak{g}_{\mathbb{R}}=\mathfrak{k}_{\mathbb{R}}+\mathfrak{p}_{\mathbb{R}}$ and complexification $\mathfrak{g}=\mathfrak{k}+\mathfrak{p}$. Following [9], we are interested in the variety $\mathbf{L}=\overline{\mathbb{O}}_{\text {min }}(\mathfrak{g}) \cap \mathfrak{p}$ in the case when $\mathbf{L} \neq\{0\}$ and the symmetric space corresponding to $\mathfrak{g}_{\mathbb{R}}$ is non-Hermitian. The main result of [9] (which preceded [1]) also exhibits "exotic" operators in $\mathcal{D}(\mathbf{L})$ of negative Euler degree. Now, there are just two such varieties $\mathbf{L}$ for $\mathfrak{g}$ classical; these being $\mathfrak{g}=\mathfrak{s l}(n) \supset \mathfrak{k}=\mathfrak{s o}(n)$ for $n \geq 3$ and $\mathfrak{g}=\mathfrak{s o}(p+q) \supset \mathfrak{k}=\mathfrak{s o}(p) \times \mathfrak{s o}(q)$ for $p \geq q \geq 3$. In both cases, we give a complete description of $\mathcal{D}(\mathbf{L})$, by identifying $\mathbf{L}$ as a categorical quotient. In particular:

Theorem 0.2. For $p \geq q \geq 3$, let $\mathfrak{g}=\mathfrak{s o}(p+q)$ have Cartan decomposition $\mathfrak{g}=\mathfrak{k}+\mathfrak{p}$ for $\mathfrak{k}=\mathfrak{s o}(p) \times \mathfrak{s o}(q)$. Set $\mathbf{L}=\overline{\mathbb{O}}_{\text {min }}(\mathfrak{g}) \cap \mathfrak{p}$. Then, under the appropriate action of $\mathrm{GL}(1)$ and its differential $\tau$ :

$$
\mathcal{D}(\mathbf{L}) \cong \frac{\left(U(\mathfrak{s o}(p+2)) / J_{0} \otimes U(\mathfrak{s o}(q+2)) / J_{0}\right)^{\mathrm{GL}(1)}}{(\tau(\mathfrak{g l}(1)))} .
$$

Finally, in Section [5 we show that the analogue of Theorem 0.1 also holds for the next to minimal orbit in $\mathfrak{s p}(2 n)$. This is, again, related to the work of Brylinski and Kostant, since we use the fact that this orbit is a shared orbit with the minimal orbit of $\mathfrak{s l}(2 n)$, in the sense of [8].

Although the results described in this paper are considerably stronger than their analogues in [1] and [9], in one respect they are considerably less satisfactory. Obviously, we are unable to prove the analogues of Theorem 0.1 for $\mathfrak{s o}(n)$ or for the Lagrangian submanifolds of the exceptional Lie algebras. Perhaps more seriously, the basic technique we use is to regard the given variety as a categorical quotient of 
a second variety and use this to construct the differential operators. Unfortunately, there is no general pattern to these second varieties, and so our arguments necessarily depend upon case by case analyses. In contrast the earlier work, particularly that of [9], provides a uniform approach for all the relevant varieties. Obviously one would like a similarly uniform approach to finding the full ring of differential operators on these varieties.

\section{Construction of some operators}

In this section we give a natural construction for the differential operators on the minimal nilpotent orbit for $\mathfrak{s l}(n)$ first described in [1]. Throughout the paper, we write $\mathbb{O}=\mathbb{O}_{\min }(\mathfrak{s l}(n))$, with closure $\overline{\mathbb{O}}$ and assume that $n \geq 2$.

Elements $a=\left(a_{i}\right) \in \mathbb{C}^{n}$ will be written as column vectors. Let $a \cdot b$ denote the scalar product on $\mathbb{C}^{n}$ and set

$$
\mathbf{X}=\left\{(a, b) \in \mathbb{C}^{n} \times \mathbb{C}^{n}: a \cdot b=0\right\} \subset \mathbf{Y}=\mathbb{C}^{n} \times \mathbb{C}^{n},
$$

and

$$
\mathbf{Z}=\left\{z=a b^{t} \text { where } a, b \in \mathbb{C}^{n}\right\} .
$$

Clearly, $\mathbf{Z}$ is the set of $n \times n$ complex matrices of rank at most one. Moreover, since $z \in \mathbf{Z}$ satisfies $z^{2}=\operatorname{tr}(z) z$, one has $z^{2}=0$ if and only if $\operatorname{tr}(z)=0$. Thus, $\overline{\mathbb{O}}$ is precisely $\{z \in \mathbf{Z}: \operatorname{tr}(z)=0\}$. Let $\mathrm{GL}(1)$ act on $\mathbf{Y}$ and $\mathbf{X}$ by $\lambda \cdot(a, b)=\left(\lambda^{-1} a, \lambda b\right)$. Thus, by classical invariant theory, $\mathbf{Z}$ is isomorphic to the variety of closed orbits $\mathbf{Y} / / \mathrm{GL}(1)$. We will always write $\mathcal{O}(\mathbf{Y})=\mathbb{C}\left[u_{i}, y_{j}: 1 \leq i, j \leq n\right]$, in which case

$$
\mathcal{O}(\mathbf{Z})=\mathcal{O}(\mathbf{Y})^{\mathrm{GL}(1)}=\mathbb{C}\left[z_{i j}: z_{i j}=u_{i} y_{j}, 1 \leq i, j \leq n\right] .
$$

Similarly, $\mathcal{O}(\mathbf{X})=\mathcal{O}(\mathbf{Y}) /\left(\sum_{i=1}^{n} u_{i} y_{i}\right)$. We will write $u_{i}$ and $y_{j}$ for the generators of $\mathcal{O}(\mathbf{X})$. Since $\mathbf{X}$ is $\mathrm{GL}(1)$-stable and $\mathrm{GL}(1)$ is reductive,

$$
\begin{aligned}
\mathcal{O}(\mathbf{X})^{\mathrm{GL}(1)} & \cong \mathcal{O}(\mathbf{Y})^{\mathrm{GL}(1)} /\left(\sum_{i} u_{i} y_{i}\right)^{\mathrm{GL}(1)} \\
& \cong \mathcal{O}(\mathbf{Y})^{\mathrm{GL}(1)} /\left(\sum_{i} u_{i} y_{i}\right) \mathcal{O}(\mathbf{Y})^{\mathrm{GL}(1)} \\
& \cong \mathcal{O}(\mathbf{Z}) / \mathcal{O}(\mathbf{Z}) T \cong \mathcal{O}(\overline{\mathbb{O}})
\end{aligned}
$$

where $T=\sum_{i=1}^{n} z_{i i}$ is the function $z \mapsto \operatorname{tr}(z)$.

Write $\operatorname{ord}(D)$ for the order of a differential operator $D$ and $D * f$ for the action of $D$ on a function $f$. Then the next lemma follows immediately from (1.1):

Lemma 1.1. (1) $\mathcal{D}(\overline{\mathbb{O}}) \cong \mathcal{D}(\mathbf{X} / / \mathrm{GL}(1))$.

(2) Restriction of functions provides a map $\rho: \mathcal{D}(\mathbf{X})^{\mathrm{GL}(1)} \longrightarrow \mathcal{D}(\mathbf{X} / / \mathrm{GL}(1))$, with kernel $\operatorname{Ker}(\rho)=\left\{\theta \in \mathcal{D}(\mathbf{X})^{\mathrm{GL}(1)}: \theta * \mathcal{O}(\mathbf{X})^{\mathrm{GL}(1)}=0\right\}$. Moreover, ord $(\rho(D)) \leq$ $\operatorname{ord}(D)$, for any operator $D \in \mathcal{D}(\mathbf{X})^{\mathrm{GL}(1)}$.

This lemma makes it easy to define differential operators on $\overline{\mathbb{O}}$. Given an indeterminate $x$, we write $\partial_{x}$ to denote the derivation $\partial / \partial x$. Let $E=\sum_{i=1}^{n} u_{i} \partial_{u_{i}}+$ $\sum_{i=1}^{n} y_{i} \partial_{y_{i}}$ denote the Euler derivation on $\mathbf{X}$ and $\Delta=\sum_{i=1}^{n} \partial_{u_{i}} \partial_{y_{i}}$ the Laplacian. Then, by [21, Proposition 2.2.2] (see also [23, Remark 3.2(v) and Corollary 5.3A]), we have

$$
\mathcal{D}(\mathbf{X})=\mathbb{C}\left\langle u_{i}, y_{j}, A_{i j}, B_{i j}, C_{i j}, E+n-1, \Phi_{j}, \Theta_{j}: 1 \leq i, j \leq n\right\rangle,
$$

where

$$
A_{i j}=-A_{j i}=y_{i} \partial_{u_{j}}-y_{j} \partial_{u_{i}}, \quad B_{i j}=-B_{j i}=u_{i} \partial_{y_{j}}-u_{j} \partial_{y_{i}}, \quad C_{i j}=y_{i} \partial_{y_{j}}-u_{j} \partial_{u_{i}}
$$


for $1 \leq i, j \leq n$, and

$$
\Phi_{j}=y_{j} \Delta-\partial_{u_{j}}(E+n-2), \quad \Theta_{j}=u_{j} \Delta-\partial_{y_{j}}(E+n-2),
$$

for $1 \leq j \leq n$. Of these, the interesting ones are the $\Phi_{j}$ and $\Theta_{j}$; they are the $z_{j}$ and $v_{j}$ of [21. Proposition 2.2.2]. The generators of $\mathcal{D}(\mathbf{X})$ have been chosen so that they generate a copy of $\mathfrak{s o}(2 n+2)$. Indeed, by [23], up to a Fourier transform, this leads to the isomorphism

$$
\mathcal{D}(\mathbf{X}) \cong U(\mathfrak{s o}(2 n+2)) / J_{0},
$$

where $J_{0}$ is a maximal ideal that, for $n \geq 3$, is the Joseph ideal.

As remarked earlier, the action of $\mathrm{GL}(1)$ on $\mathcal{D}(\mathbf{X})$ is defined by $\lambda \cdot u_{i}=\lambda u_{i}$ and $\lambda \cdot y_{i}=\lambda^{-1} y_{i}$, whence $\lambda \cdot \partial_{u_{i}}=\lambda^{-1} \partial_{u_{i}}$ and $\lambda \cdot \partial_{y_{i}}=\lambda \partial_{y_{i}}$. For future reference, notice that in the notation of the introduction,

$$
\tau(\mathfrak{g l}(1))=\mathbb{C} \Omega \quad \text { where } \quad \Omega=-\sum_{i=1}^{n} C_{i i}=\sum_{i=1}^{n}\left(u_{i} \partial_{u_{i}}-y_{i} \partial_{y_{i}}\right) .
$$

It is clear that

$$
\begin{array}{ll}
\lambda \cdot \Delta=\Delta, & \lambda \cdot E=E, \\
\lambda \cdot A_{i j}=\lambda^{-2} A_{i j}, & \lambda \cdot B_{i j}=\lambda^{2} B_{i j}, \quad \lambda \cdot C_{i j}=C_{i j}, \\
\lambda \cdot \Phi_{j}=\lambda^{-1} \Phi_{j}, & \lambda \cdot \Theta_{j}=\lambda \Theta_{j} .
\end{array}
$$

Consequently, $\mathcal{D}(\mathbf{X})^{\mathrm{GL}(1)}$ is generated by the elements

$$
\begin{array}{lllll}
u_{i} y_{j} & \Theta_{i} \Phi_{j} & C_{i j} & E, & \\
A_{i j} B_{k \ell} & u_{k} u_{\ell} A_{i j} & y_{k} y_{\ell} B_{i j} & \Theta_{k} \Theta_{\ell} A_{i j} & \Phi_{k} \Phi_{\ell} B_{i j}, \\
u_{i} \Phi_{j} & y_{i} \Theta_{j} & u_{k} \Theta_{\ell} A_{i j} & y_{k} \Phi_{\ell} B_{i j} . &
\end{array}
$$

The elements of $\mathcal{D}(\overline{\mathbb{O}})$ that particularly interest us are the $\rho\left(\Phi_{i} \Theta_{j}\right)$. Let $E_{z}$ denote the Euler derivation on $\overline{\mathbb{O}}$; thus $E_{z} * z_{i j}=z_{i j}$ for all $i$ and $j$. Since $\rho(E) * z_{i j}=$ $E * z_{i j}=2 z_{i j}$ we see that $\rho(E)=2 E_{z}$. From the equation

$$
-2 \rho\left(\Phi_{i} \Theta_{j}\right)=\rho\left(\left[E, \Phi_{i} \Theta_{j}\right]\right)=\left[\rho(E), \rho\left(\Phi_{i} \Theta_{j}\right)\right]=2\left[E_{z}, \rho\left(\Phi_{i} \Theta_{j}\right)\right],
$$

we see that $\rho\left(\Phi_{i} \Theta_{j}\right)$ has Euler degree -1 and order at most 4 .

Proposition 1.2. The operator $-\frac{1}{4} \rho\left(\Phi_{n} \Theta_{1}\right)$ is the element $D_{0}$ of [1, Proposition 4.2.3]. Moreover, the elements $\rho\left(\Phi_{i} \Theta_{j}\right)$, for $1 \leq i, j \leq n$, span the $\mathfrak{s l}(n)$ submodule $\mathcal{A}_{-1}$ of $\mathcal{D}(\overline{\mathbb{O}})$ defined in that paper. This module is isomorphic to the adjoint representation of $\mathfrak{s l}(n)$.

Remark 1.3. We will not define $D_{0}$ and $\mathcal{A}_{-1}$ as they are complicated; indeed, the whole point of our approach is that we do not need to write them out explicitly in terms of the $z_{i j}$.

Proof. For notational reasons, it is easier to consider $\mathbb{O}$ as an orbit in $\mathfrak{g l}(n)$ rather than in $\mathfrak{s l}(n)$. Let $E_{a b}$ denote the standard basis of $\mathfrak{g l}(n)$; thus $z_{i j}=u_{i} y_{j}$ are the functions on $\overline{\mathbb{O}}$ induced by the coordinate functions on the $E_{i j}$. The action of $\operatorname{GL}(n)$ on $\overline{\mathbb{O}}$ induces a Lie algebra homomorphism $\eta: \mathfrak{g l}(n) \rightarrow \operatorname{Der} \mathcal{O}(\overline{\mathbb{O}})$ written $\xi \mapsto \eta^{\xi}$. Thus,

$$
\eta^{E_{a b}} * z_{i j}\left(E_{u v}\right)=z_{i j}\left(\left[E_{u v}, E_{a b}\right]\right)=z_{i j}\left(\delta_{a v} E_{u b}-\delta_{b u} E_{a v}\right),
$$




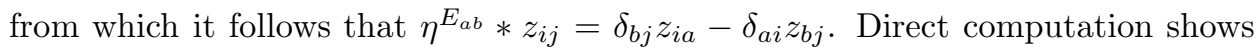
that this equals $C_{a b} * z_{i j}$ and so

$$
\rho\left(C_{a b}\right)=\eta^{E_{a b}} \quad \text { for } \quad 1 \leq a, b \leq n .
$$

Similarly, one finds that

$$
\left[C_{k k}, \Phi_{i} \Theta_{j}\right]=\left(\delta_{k i}-\delta_{k j}\right) \Phi_{i} \Theta_{j} \quad \text { for } \quad 1 \leq i, j, k \leq n,
$$

and so, in Bourbaki's notation $\rho\left(\Phi_{i} \Theta_{j}\right)$ has weight $\varepsilon_{i}-\varepsilon_{j}$.

It follows from the computations of the last paragraph that $z_{n 1}=u_{n} y_{1}$ is a highest weight vector of the $\mathfrak{g l}(n)$-module $\mathcal{O}(\overline{\mathbb{O}})_{1}=\sum \mathbb{C} z_{i j} \subset \mathcal{O}(\overline{\mathbb{O}})$ and hence that $z_{n 1}=f_{e}$, in the notation of [1, Section 3]. Since $\Delta *\left(u_{n}^{a} y_{1}^{b}\right)=0$, for all $a, b$, we see that

$$
\begin{aligned}
\Phi_{n} \Theta_{1} *\left(u_{n}^{r} y_{1}^{r}\right) & =\Phi_{n} *\left(-(2 r+n-2) r u_{n}^{r} y_{1}^{r-1}\right) \\
& =(2 r+n-2)(2 r+n-3) r^{2} u_{n}^{r-1} y_{1}^{r-1} .
\end{aligned}
$$

Note that, as $r$ appears as a polynomial of degree 4 in this equation, this also shows that $\rho\left(\Phi_{n} \Theta_{1}\right)$ has order $\geq 4$, and hence equal to 4 . Comparing this equation with [1, Proposition 4.2.3] shows that $\rho\left(\Phi_{n} \Theta_{1}\right)=-4 D_{0}$. By computing $\left[C_{a b}, \Phi_{i} \Theta_{j}\right]$ one finds that $\sum_{i, j} \mathbb{C} \rho\left(\Phi_{i} \Theta_{j}\right)$ is, indeed, isomorphic to the adjoint representation of $\mathfrak{s l}(n)$ under $\eta$ (the element $\sum \rho\left(\Phi_{i} \Theta_{i}\right)$ is zero).

\section{Complete Description of $\mathcal{D}(\overline{\mathbb{O}})$}

The aim of this section is to prove Theorem 0.1 from the introduction. The idea behind the proof is similar to the one used in 23] and 24]: The major step is to show that $A=\operatorname{Im}(\rho)$ satisfies $\operatorname{GK} \operatorname{dim} \mathcal{D}(\overline{\mathbb{O}}) / A \leq \operatorname{GKdim} A-2$, after which the result follows from standard Cohen-Macaulay type arguments. In this section we need to assume that $n \geq 3$ as the analogue of Theorem 0.1 fails for $n=2$. We discuss that case in the next section.

Lemma 2.1. Let $A=\operatorname{Im}(\rho)$. If $p \in \mathbb{O}$, we write $\mathcal{O}(\overline{\mathbb{O}})_{p}$ for the local ring of $\overline{\mathbb{O}}$ at $p$. Then, $\operatorname{Der} \mathcal{O}(\overline{\mathbb{O}})_{p} \subset A_{p}=A \otimes_{\mathcal{O}(\overline{\mathbb{O}})} \mathcal{O}(\overline{\mathbb{O}})_{p}$.

Proof. As $\mathbb{O}$ is an orbit, it suffices to prove the lemma at any prespecified point $p \in \mathbb{O}$, and we choose $p=E_{1 n}$. One has $C_{i j} * z_{a b}=\delta_{j b} z_{a i}-\delta_{a i} z_{j b}$, for all $i, j, a, b$. It follows easily that the operators $C_{j 1}$ and $C_{n k}$ for $j<n$, and $k \leq n$ provide enough derivations to generate $\operatorname{Der} \mathcal{O}(\overline{\mathbb{O}})_{p}$. More precisely, we define

$$
\begin{aligned}
d_{1 j} & =z_{1 n}^{-1} C_{n j}, & & 1 \leq j \leq n-1, \\
d_{i n} & =-z_{1 n}^{-1} C_{i 1}+z_{11} z_{1 n}^{-1} d_{11}, & & 2 \leq i \leq n-1, \\
d_{1 n} & =z_{1 n}^{-1} C_{n n}-\sum_{i=2}^{n-1} z_{1 n}^{-1} z_{i n} d_{i n} . & &
\end{aligned}
$$

Note that, as $z_{i j}\left(E_{k l}\right)=\delta_{i k} \delta_{j l}, B=\left\{z_{11}, \ldots, z_{1 n-1}, z_{1 n}-1, z_{2 n}, \ldots, z_{n-1 n}\right\}$ is a minimal generating set of the maximal ideal of the local ring $\mathcal{O}(\overline{\mathbb{O}})_{E_{1 n}}$. Then, $\left\{d_{i j}\right\}$ is a "dual basis" of $B$.

Observe that $\mathbb{O}$ is stable under the homotheties $x \mapsto \lambda x, \lambda \in \mathbb{C}^{*}$. Thus the coordinate ring $\mathcal{O}(\mathbb{O})$ is naturally graded and we denote its augmentation ideal by $P$. 
Corollary 2.2. (i) For all $p \in \mathbb{O}, A_{p}=\mathcal{D}(\overline{\mathbb{O}})_{p}$. In particular, $A$ and $\mathcal{D}(\overline{\mathbb{O}})$ have the same division ring of fractions.

(ii) Let $d \in \mathcal{D}(\overline{\mathbb{O}})$ and $M=A d+A / A$. Then, $M_{p}=0$ for all $p \in \mathbb{O}$ and hence $P^{t} d \in A$, for some $t$.

We let $\operatorname{GKdim}(R)$ denote the Gelfand-Kirillov dimension of a $\mathbb{C}$-algebra $R$. Let $R$ be filtered by $\left\{R^{m}\right\}$ such that $\operatorname{gr}(R)=\bigoplus_{m} R^{m} / R^{m-1}$ is an affine commutative domain. Denote by $V$ the variety corresponding to $\operatorname{gr}(R)$. Assume that $G$ is a reductive algebraic group acting regularly on $R$ and preserving the filtration. Then

$$
\operatorname{GKdim}\left(R^{G}\right) \leq \operatorname{GKdim}(R)-\max \{\operatorname{dim} G . v: v \in V\} .
$$

Indeed, since $G$ is reductive, we have $\operatorname{gr}(R)^{G}=\operatorname{gr}\left(R^{G}\right)$ and (2.1) follows from [16. II.4.3.E].

Recall that $\tau: \mathfrak{g r}(1) \rightarrow \mathcal{D}(\mathbf{X})$ is the differential of the action of GL(1) on $\mathcal{D}(\mathbf{X})$ and that, by (1.4),$\tau(\mathfrak{g l}(1))=\mathbb{C} \Omega$. By its very definition, $\tau(\mathfrak{g l}(1)) \subset \operatorname{Ker}(\rho)$.

Lemma 2.3. (1) $\operatorname{GKdim} \mathcal{D}(\mathbf{X})=\operatorname{GKdim} U(\mathfrak{s o}(2 n+2)) / J_{0}=4 n-2$.

(2) $\operatorname{GKdim} A=4 n-4$.

(3) $\operatorname{GKdim} \mathcal{D}(\mathbf{X})^{\mathrm{GL}(1)}=4 n-3$ and hence $\operatorname{Ker}(\rho)$ has height one.

Proof. Part (1) is immediate from [21 Lemma 3.2 and Proposition 3.3], while part (2) follows from Corollary 2.2 combined with the fact that $\mathbb{O}$ has dimension $2 n-2$. In order to prove $(3)$, we note that $\operatorname{Ker}(\rho) \neq 0$, since it contains the nonzero element $\Omega$. Since $\mathcal{D}(\mathbf{X})^{\mathrm{GL}(1)}$ is a domain, [15, Proposition 3.15] implies that $\operatorname{GKdim}\left(\mathcal{D}(\mathbf{X})^{\mathrm{GL}(1)}\right) \geq \mathrm{GKdim}(A)+1=4 n-3$. On the other hand, since the $\mathrm{GL}(1)$ action is not finite on $\mathcal{D}(\mathbf{X})$, we have $\operatorname{GKdim}\left(\mathcal{D}(\mathbf{X})^{\mathrm{GL}(1)}\right) \leq \operatorname{GKdim}(\mathcal{D}(\mathbf{X}))-1=$ $4 n-3$ by (2.1).

Notation 2.4. For the next few results we wish to study the structure of modules over $\mathfrak{s o}(2 n+2)$ and here we establish the pertinent notation. For the rest of the section, we define $\mathfrak{g}=\mathfrak{s o}(2 n+2)$. In the notation of [23, Remarks 3.2(v)], for $\mathfrak{g}$ of type $D_{n+1}$, we let $\mathfrak{p}$ be the parabolic $\mathfrak{p}_{1}$ and the corresponding decomposition $\mathfrak{g}=\mathfrak{r}^{+} \oplus \mathfrak{m} \oplus \mathfrak{r}^{-} \supset \mathfrak{p}=\mathfrak{m} \oplus \mathfrak{r}^{+}$with $\mathfrak{m}=[\mathfrak{m}, \mathfrak{m}] \oplus \mathbb{C} H \cong \mathfrak{s o}(2 n) \oplus \mathbb{C} H$. Set $\mathfrak{p}^{-}=$ $\mathfrak{m} \oplus \mathfrak{r}^{-}$. Recall that the generators of $\mathcal{D}(\mathbf{X})$ in (1.2) were chosen to generate a copy of $\mathfrak{s o}(2 n+2)$. (The description of $\mathfrak{s o}(2 n+2) \subset \mathcal{D}(\mathbf{X})$ in [21] differs from that of [23] by a Fourier transform which interchanges $\mathfrak{r}^{-}$and $\mathfrak{r}^{+}$.) By [21, Proposition 2.2.2], this parabolic decomposition is chosen so that $\mathfrak{r}^{-}$has basis $\left\{u_{i}, y_{j}\right\}, \mathfrak{r}^{+}$has basis $\left\{\Theta_{i}, \Phi_{j}\right\}$, and $[\mathfrak{m}, \mathfrak{m}]$ has basis $\left\{A_{i j}, B_{i j}, C_{i j}\right\}$, while $H=E+n-1$. Note that $\Omega=-\sum C_{i i} \in[\mathfrak{m}, \mathfrak{m}]$. We let $\mathfrak{q}$ denote the subalgebra generated by $H$ and the $C_{i j}$; this is isomorphic to $\mathfrak{g l}(n) \oplus \mathbb{C} H$.

Let $\mathbf{O}_{\min }=\mathbb{O}_{\min }(\mathfrak{s o}(2 n+2))$ denote the minimal nilpotent orbit of $\mathfrak{g}$. It follows from [23] that $\mathbf{X}$ is an irreducible component of $\overline{\mathbf{O}}_{\text {min }} \cap \mathfrak{r}^{+}$and $\mathcal{D}(\mathbf{X}) \cong U(\mathfrak{g}) / J_{0}$. This implies that $\mathcal{D}(\mathbf{X})$ has two filtrations; one induced from the standard filtration on $U(\mathfrak{g})$ and the other being that given by order of differential operator. We emphasize that, except where it is explicitly stated to the contrary, we will always use the former filtration. Since $\operatorname{gr}\left(J_{0}\right)$ is prime [11, Theorem V.2], it follows from 23] that $\mathcal{D}(\mathbf{X})$ has associated graded ring isomorphic to $\mathcal{O}\left(\overline{\mathbf{O}}_{\text {min }}\right)$.

Lemma 2.5. Let $R$ be a $\mathbb{C}$-algebra and $c \in R$ an ad-nilpotent, regular element. Write $\mathcal{C}=\left\{c^{n}\right\}$ and $R_{\mathcal{C}}$ for the localization of $R$ at $\mathcal{C}$. If $M$ is a right $R$-module, then $\operatorname{GKdim}_{R}(M)=\max \left\{\operatorname{GKdim}_{R_{\mathcal{C}}}\left(M_{\mathcal{C}}\right), \operatorname{GKdim}_{R}(T)\right\}$, where $T=\left\{m \in M: m c^{r}=0\right.$ for some $r \geq 1$ \}. 
Proof. This is implicit in [3] and proved in detail in [25, Theorem 3.2].

The hard work in proving Theorem 0.1 involves estimating $\operatorname{GKdim}(A / A P)$, in the notation of Lemma 2.1. We obtain this estimate by pulling the question back to one about $\mathcal{D}(\mathbf{X})$-modules, since this allows us to use the geometry of $\mathfrak{s o}(2 n+2)$. The details are given in the next several lemmas.

Lemma 2.6. Assume that $n \geq 3$. Set $I=\sum_{i=1}^{n} \mathcal{D}(\mathbf{X}) u_{i}+\mathcal{D}(\mathbf{X})(\Omega-\lambda)$, for some $\lambda \in \mathbb{C}$ and let $J=I+\sum_{j=1}^{n} \mathcal{D}(\mathbf{X}) y_{j}$. If $\operatorname{GKdim}(\mathcal{D}(\mathbf{X}) / J) \leq \operatorname{GKdim}(\mathcal{D}(\mathbf{X}))-3$, then $\operatorname{GKdim}(\mathcal{D}(\mathbf{X}) / I) \leq \operatorname{GKdim}(\mathcal{D}(\mathbf{X}))-3$.

Proof. Set $I_{k}=I_{k, \lambda}=I+\sum_{j=1}^{k-1} \mathcal{D}(\mathbf{X}) y_{j}$; thus $I_{n+1}=J$ and $I_{1}=I$. Therefore, by induction, assume that $\operatorname{GKdim}\left(\mathcal{D}(\mathbf{X}) / I_{k+1}\right) \leq \operatorname{GKdim}(\mathcal{D}(\mathbf{X}))-3$, for some $k \leq n$. We need to prove that $\operatorname{GKdim}\left(\mathcal{D}(\mathbf{X}) / I_{k}\right) \leq \operatorname{GKdim}(\mathcal{D}(\mathbf{X}))-3$.

Let $c=y_{k}$, set $\mathcal{C}=\left\{c^{r}\right\}$ and consider $S=\mathcal{D}(\mathbf{X})_{\mathcal{C}}=\mathcal{D}\left(\mathcal{O}(\mathbf{X})_{\mathcal{C}}\right)^{\text {. Clearly, }}$

$$
\mathcal{O}(\mathbf{X})_{\mathcal{e}} \cong \mathbb{C}\left[u_{i}, y_{j}, y_{k}^{-1}: 1 \leq i, j \leq n, \text { but } i \neq k\right]
$$

is a localization of a polynomial ring in $2 n-1$ variables and so $S$ is a localization of the corresponding Weyl algebra. By considering the action of $\Omega$ on these generators, one finds that, as an element of $S,-c^{-1} \Omega$ is the derivation $\delta=\partial_{y_{k}}-\sum_{i \neq k} c^{-1}\left(u_{i} \partial_{u_{i}}-y_{j} \partial_{y_{j}}\right)$. Since $I$ contains the generators $\left\{u_{i}: i \neq k\right\}$ and $\delta$, it follows easily that $\operatorname{GKdim}\left(S / S I_{k}\right) \leq \operatorname{GKdim}(S / S I)=\operatorname{GKdim}(S)-n \leq$ $\operatorname{GKdim}(S)-3$. Set $T=\left\{t \in \mathcal{D}(\mathbf{X}): y_{k}^{r} t \in I_{k}\right.$ for some $\left.r \geq 1\right\}$. Then, by Lemma 2.5 . $\operatorname{GKdim}\left(\mathcal{D}(\mathbf{X}) / I_{k}\right) \leq \operatorname{GKdim}(\mathcal{D}(\mathbf{X}))-3$ will hold provided that $\operatorname{GKdim}\left(T / I_{k}\right) \leq$ $\operatorname{GKdim}(\mathcal{D}(\mathbf{X}))-3$.

Let $t \in T$, say, with $y_{k}^{r} t \in I_{k}$. Since the $y_{j}$ and $u_{i}$ act ad-nilpotently on $\mathcal{D}(\mathbf{X})$, we may pick $r$ such that $u_{i}^{r} t \in I_{k}$ and $y_{j}^{r} t \in I_{k}$, for $1 \leq i \leq n$ and $1 \leq j<$ $k$. Also, by Notation $2.4 \Omega$ is an element of $\mathfrak{s o}(2 n+2)$ and so acts ad-finitely on $\mathcal{D}(\mathbf{X}) \cong U(\mathfrak{s o}(2 n+2)) / J_{0}$. Thus, $p(\Omega) t \in I_{k}$, for some nonzero polynomial $p(\Omega) \in \mathbb{C}[\Omega]$. Hence, $L t \subset I_{k}$, for $L=\mathcal{D}(\mathbf{X}) u_{1}^{r}+\cdots+\mathcal{D}(\mathbf{X}) y_{k}^{r}+\mathcal{D}(\mathbf{X}) p(\Omega)$. Since $u_{i} \Omega=(\Omega-1) u_{i}$ and $y_{j} \Omega=(\Omega+1) y_{j}$, a routine induction shows that $\mathcal{D}(\mathbf{X}) / L$ has a finite composition series where each of the subfactors is a factor of $I_{k+1, \mu}$, for some scalars $\mu$. (To begin this induction, note that $L^{\prime} u_{1}^{r-1} \subset L$, where $L^{\prime}=\mathcal{D}(\mathbf{X}) u_{1}+\mathcal{D}(\mathbf{X}) u_{2}^{r}+\cdots+\mathcal{D}(\mathbf{X}) y_{k}^{r}+\mathcal{D}(\mathbf{X}) p(\Omega+r-1)$.) But, by induction, $\operatorname{GKdim}\left(\mathcal{D}(\mathbf{X}) / I_{k+1, \mu}\right) \leq \operatorname{GKdim}(\mathcal{D}(\mathbf{X}))-3$, for any such $\mu$. Thus, $\operatorname{GKdim}\left(T / I_{k}\right) \leq \operatorname{GKdim}(\mathcal{D}(\mathbf{X}))-3$, as required.

In the sequel we will identify $\mathfrak{g}$ with $\mathfrak{g}^{*}$ through the Killing form $\kappa$. Then, $S(\mathfrak{g}) \equiv \mathcal{O}(\mathfrak{g})$ and $X \in \mathfrak{g}$ identifies with $\kappa\left(X,,_{-}\right) \in \mathfrak{g}^{*}$. If $I \subset S(\mathfrak{g})$ is an ideal, we denote its variety of zeroes by $\mathcal{V}(I) \subset \mathfrak{g} \equiv \mathfrak{g}^{*}$.

Lemma 2.7. In the notation of Lemma 2.6.

$$
\operatorname{GKdim}(\mathcal{D}(\mathbf{X}) / I) \leq \operatorname{GKdim}(\mathcal{D}(\mathbf{X}))-3 .
$$

Proof. We work in the associated graded $\operatorname{ring} R=\operatorname{gr}(\mathcal{D}(\mathbf{X})) \cong \mathcal{O}\left(\overline{\mathbf{O}}_{\text {min }}\right)$; thus by Lemma 2.6 it suffices to prove that $\operatorname{GKdim}(R / \tilde{J}) \leq \operatorname{GKdim}(R)-3$, where $\tilde{J}=$ $\sum_{1 \leq i \leq n}\left(R u_{i}+R y_{i}\right)+R \Omega \subset \operatorname{gr}(J)$, and we have identified $\Omega$ with its image in $R$. Set $L=\sum_{1 \leq i \leq n} R u_{i}+R y_{i}$. Thus, by Notation 2.4, $L$ defines the subvariety $\overline{\mathbf{O}}_{\min } \cap \mathfrak{p}^{-}$ of $\overline{\mathbf{O}}_{\text {min }}$ and, by [23. Theorem 4.7], one has $\operatorname{dim}\left(\overline{\mathbf{O}}_{\min } \cap \mathfrak{p}^{-}\right) \leq \operatorname{dim}\left(\overline{\mathbf{O}}_{\text {min }}\right)-2$. The proof is now similar to that of analogous results in [23]. 
The identification $R=\mathcal{O}\left(\overline{\mathbf{O}}_{\text {min }}\right)$ is induced from identifying $\operatorname{gr}(U(\mathfrak{g}))$ with $\mathcal{O}(\mathfrak{g})$. As such, we may write $\mathfrak{g}=F \oplus \mathbb{C} \Omega$, where $F$ is the zero set of $\Omega$. As in Notation 2.4 $\Omega \in \mathfrak{m}_{1}=[\mathfrak{m}, \mathfrak{m}]$. Now let $V$ be an irreducible subvariety of $\overline{\mathbf{O}}_{\min } \cap \mathfrak{p}^{-}$. We need to prove that $\operatorname{dim}(V \cap F) \leq \operatorname{dim}\left(\overline{\mathbf{O}}_{\text {min }}\right)-3$. If $V \subset \overline{\mathbf{O}}_{\min } \cap \mathfrak{r}^{-}$, then [23. Table 3.1] implies that $\operatorname{dim}(V) \leq 2 n-1$, while $\operatorname{dim}\left(\overline{\mathbf{O}}_{\text {min }}\right)=4 n-2$. Since $n \geq 3$, this implies that $\operatorname{dim}(V \cap F) \leq \operatorname{dim} V \leq \operatorname{dim}\left(\overline{\mathbf{O}}_{\min }\right)-3$.

Thus, we are left with the case when $V \not \subset \overline{\mathbf{O}}_{\text {min }} \cap \mathfrak{r}^{-}$. We now follow the proof of 23. Claim 4.4]. Pick $W=W_{1}+\mu H+W_{-} \in V$ where $W_{1} \in \mathfrak{m}_{1}, W_{-} \in \mathfrak{r}^{-}$and $W_{1}+\mu H \neq 0$. By the argument of [23, (4.4)] this implies that $W_{1} \neq 0$. Since $\mathfrak{m}_{1}$ is simple, there exists $g$ in the adjoint group of $\mathfrak{m}_{1}$, such that $g . W_{1}=\lambda \Omega+Z$ where $\lambda \neq 0$ and $Z \in F$. As in the proof of [23, Claim 4.4], we may replace $W$ by $g . W$ and assume that $W=\lambda \Omega+W^{\prime} \in \mathbf{O}_{\min } \cap \mathfrak{p}^{-}$with $W^{\prime} \in F$ and $\lambda \neq 0$. Thus, this proves that

$$
\operatorname{dim}(V \cap \mathcal{V}(\tilde{J}))<\operatorname{dim}(V) \leq \operatorname{dim}\left(\overline{\mathbf{O}}_{\min } \cap \mathfrak{p}^{-}\right) \leq \operatorname{dim}\left(\overline{\mathbf{O}}_{\min }\right)-2,
$$

as required.

We remark that the inequality in Lemma 2.7 is actually an equality, which is why the proof is fairly delicate. One can rephrase (and prove) the result more geometrically as the assertion that $\operatorname{dim} \overline{\mathbf{O}}_{\min } \cap \mathcal{V}\left(\left(u_{1}, \ldots, u_{n}, \Omega\right)\right)=4 n-5$.

Recall the action of $\mathrm{GL}(1)$ on $\mathfrak{g}=\mathfrak{s o}(2 n+2)$ given in (1.5).

Lemma 2.8. Let $\mathfrak{q}$ be defined as in Notation 2.4. Let I be an ideal of $S(\mathfrak{g})$ that is stable under the GL(1)-action, and assume that

$$
\operatorname{dim}(S(\mathfrak{g}) / I)^{\mathrm{GL}(1)}=\operatorname{dim} S(\mathfrak{g}) / I .
$$

Then, $\mathcal{V}(I)$ is contained in $\mathfrak{q}$.

Proof. Note that $\mathfrak{q}=\mathfrak{g}^{\mathrm{GL}(1)}$. Let $\mathfrak{s}$ denote the sum of the nontrivial representations of $\mathrm{GL}(1)$ in $\mathfrak{g}$; thus $\mathfrak{g}=\mathfrak{s} \oplus \mathfrak{q}$. By (2.2), the $\mathrm{GL}(1)$ action on $S(\mathfrak{g}) / I$ must factor through a finite action and so, for all homogeneous elements $X \in S(\mathfrak{g})$, there must exist $i \geq 1$ such that $\lambda^{i} \cdot X \equiv X$ modulo $I$, for all $\lambda \in \mathbb{C}^{*}$. However, if $x \in \mathfrak{s}$, (1.5) implies that $\lambda \cdot x=\lambda^{\epsilon} x$, where $\epsilon \in\{ \pm 1, \pm 2\}$. Thus, for all $x \in \mathfrak{s}$, there exists $m$ such that $x^{m} \in I$. In other words, $\mathcal{V}(I) \subset \mathfrak{q}$.

Lemma 2.9. Write $P$ for the augmentation ideal of $\mathcal{O}(\mathbf{X})^{\mathrm{GL}(1)}=\mathcal{O}(\overline{\mathbb{O}})$ and let $M=\mathcal{D}(\mathbf{X})^{\mathrm{GL}(1)} \Omega+\mathcal{D}(\mathbf{X})^{\mathrm{GL}(1)} P$. Then,

$$
\operatorname{GKdim}\left(\mathcal{D}(\mathbf{X})^{\mathrm{GL}(1)} / M\right) \leq \operatorname{GKdim}\left(\mathcal{D}(\mathbf{X})^{\mathrm{GL}(1)}\right)-3 .
$$

Proof. Recall that $\operatorname{GKdim}\left(\mathcal{D}(\mathbf{X})^{\mathrm{GL}(1)}\right)=4 n-3$ and $\operatorname{GKdim}(\mathcal{D}(\mathbf{X}))=4 n-2$, by Lemma 2.3. We first estimate $\operatorname{GKdim}(N)$, where $N=\mathcal{D}(\mathbf{X}) /(\mathcal{D}(\mathbf{X}) P+\mathcal{D}(\mathbf{X}) \Omega)$. As in the proof of Lemma 2.6, $N$ has a composition series where each subfactor is a factor of either

$$
M=\mathcal{D}(\mathbf{X}) /\left(\sum_{i=1}^{n} \mathcal{D}(\mathbf{X}) u_{i}+\mathcal{D}(\mathbf{X})(\Omega-\mu)\right),
$$

or $M^{\prime}=\mathcal{D}(\mathbf{X}) /\left(\sum_{i=1}^{n} \mathcal{D}(\mathbf{X}) y_{i}+\mathcal{D}(\mathbf{X})(\Omega-\mu)\right)$, for some $\mu \in \mathbb{C}$. By Lemma 2.7 $\operatorname{GKdim}(M) \leq \operatorname{GKdim}(\mathcal{D}(\mathbf{X}))-3$, and so $\operatorname{GKdim}(N) \leq \operatorname{GKdim}(\mathcal{D}(\mathbf{X}))-3=4 n-5$.

It remains to prove that

$$
\operatorname{GKdim}\left(N^{\mathrm{GL}(1)}\right) \leq \operatorname{GKdim}\left(\mathcal{D}(\mathbf{X})^{\mathrm{GL}(1)}\right)-3=4 n-6 .
$$


By the last paragraph this holds if $\operatorname{GKdim}\left(N^{\mathrm{GL}(1)}\right) \leq \operatorname{GKdim}(N)-1$. Let $I$ denote the associated graded ideal, in $\mathcal{O}\left(\overline{\mathbf{O}}_{\text {min }}\right)$, of $\mathcal{D}(\mathbf{X}) P+\mathcal{D}(\mathbf{X})(\Omega-\lambda)$. If $\operatorname{GKdim}\left(N^{\mathrm{GL}(1)}\right)=\operatorname{GKdim}(N)$, then Lemma 2.8 implies that $\mathcal{V}(I) \subset \mathfrak{q} \cap \overline{\mathbf{O}}_{\text {min. }}$. Now, $\overline{\mathbf{O}}_{\text {min }}$ consists of matrices of rank at most two and square zero. Hence, so does $\overline{\mathbf{O}}_{\text {min }} \cap \mathfrak{q}$. By Notation $2.4 \mathfrak{q} \cong \mathfrak{g l}(n) \oplus \mathbb{C} H$, where $H$ is semisimple. Thus, [24, Lemma II.6.7] implies that $\operatorname{dim} \overline{\mathbf{O}}_{\min } \cap \mathfrak{q} \leq 4 n-8$, as desired.

As an application of Lemma 2.9 we show that $\operatorname{Ker}(\rho)=(\Omega)$. One way of proving this is to use idealizers to pull the result down from $\mathcal{D}(\mathbf{Y})^{\mathrm{GL}(1)}$, where the corresponding result holds by 26]. However, we will use a slightly different method, which will also be used in Section 4. The next two lemmas provide the method of attack. Given $f \in \mathcal{O}(\mathbf{W})$, for a variety $\mathbf{W}$, we write $\mathcal{D}(\mathbf{W})_{f}$ for the localization of $\mathcal{D}(\mathbf{W})$ at the powers of $f$; equivalently, it is isomorphic to $\mathcal{D}\left(\mathcal{O}(\mathbf{W})_{f}\right)$.

Lemma 2.10. (i) The fixed ring $F=U(\mathfrak{s o}(2 n+2))^{\mathrm{GL}(1)}$ is Auslander-Gorenstein.

(ii) F satisfies the following Cohen-Macaulay condition: If $M \neq 0$ is a finitely generated $F$-module, set $\mathrm{j}_{F}(M)=\min \left\{j: \operatorname{Ext}_{F}^{j}(M, F) \neq 0\right\}$. Then

$$
\operatorname{GKdim}(M)+\mathrm{j}_{F}(M)=\operatorname{GKdim}(F) \text {. }
$$

Proof. The ring $F$ has associated graded ring $\mathcal{O}(\mathfrak{s o}(2 n+2))^{\mathrm{GL}(1)}$ which is Gorenstein by [30, Theorem 6.7]. The result now follows from [20, Theorem 2.2].

Lemma 2.11. Suppose that GL(1) acts on an irreducible affine variety $\mathbf{W}$, with differential $\tau$. Write $\tau(\mathfrak{g l}(1))=\mathbb{C} \Theta$ and let $\rho: \mathcal{D}(\mathbf{W})^{\mathrm{GL}(1)} \rightarrow \mathcal{D}(\mathbf{W} / / \mathrm{GL}(1))$ denote the natural map. Assume that:

(i) The ring $D=\mathcal{D}(\mathbf{W})^{\mathrm{GL}(1)}$ is filtered so that the associated graded ring $\mathrm{gr} D$ is a finitely generated, commutative, Cohen-Macaulay domain.

(ii) There exists an ideal $K=\sum_{i} f_{i} \mathcal{O}(\mathbf{W})^{\mathrm{GL}(1)}$ such that, for each $i$, the induced map $\rho_{i}: D_{f_{i}} \rightarrow \mathcal{D}(\mathbf{W} / / \mathrm{GL}(1))_{f_{i}}$ has kernel $D_{f_{i}} \Theta$.

(iii) If $\widetilde{K}=D \Theta+D K$, then $\operatorname{GKdim}(D / \widetilde{K}) \leq \operatorname{GKdim}(D)-2$.

Then, $\operatorname{Ker}(\rho)=D \Theta$.

Proof. As GL(1) is abelian, $\Theta$ is central in $D$. Let $\phi \in \operatorname{Ker}(\rho)$ and suppose that $\phi \notin D \Theta$. For each $i, \phi \in \operatorname{Ker}\left(\rho_{i}\right)$ and so, by hypothesis (ii), there exists an integer $k$ such $f_{i}^{k} \phi \subseteq D \Theta$. In other words, $L \phi \subseteq D \Theta$, where $L=D \Theta+D K^{\ell}$, for some integer $\ell$. An easy induction using (iii) shows that $\operatorname{GKdim}(D / L) \leq \operatorname{GKdim}(D)-2$.

For any left ideal $M$ of $D$, give $M$ and $D / M$ the induced filtrations from that of $D$. Then, $\operatorname{gr} D / \operatorname{gr} L \cong \operatorname{gr}(D / L)$ and so $\operatorname{GKdim}(\operatorname{gr} D / \operatorname{gr} L) \leq \operatorname{GKdim}(\operatorname{gr} D)-2$. Now gr $D$ is Cohen-Macaulay with pure dimension, in the sense that

$$
\operatorname{GKdim}(\operatorname{gr} D / P)+\operatorname{ht} P=\operatorname{GKdim}(\operatorname{gr} D)
$$

for any prime ideal $P$. It follows from [5. Corollary 2.1.4] that $\mathrm{j}_{\operatorname{gr} D}(\operatorname{gr}(D / L)) \geq 2$. Hence, by [2, Proposition 3.1], $\mathrm{j}_{D}(D / L) \geq 2$; thus $\operatorname{Ext}_{D}^{1}(D / L, D)=0$. Since $(D \Theta+D \phi) / D \Theta$ is a homomorphic image of $D / L$ and $D$ is a domain, the usual long exact sequence in cohomology implies that

$$
0=\operatorname{Ext}_{D}^{1}(D \Theta+D \phi / D \Theta, D)=\operatorname{Ext}_{D}^{1}(D \Theta+D \phi / D \Theta, D \Theta) .
$$

But, $D \Theta+D \phi$ is an essential extension of $D \Theta$ and so $\operatorname{Ext}_{D}^{1}(D \Theta+D \phi / D \Theta, D \Theta) \neq 0$, giving the required contradiction.

We now apply these results to the variety $\mathbf{X}$. 
Proposition 2.12. $\operatorname{Ker}(\rho)=\tau(\mathfrak{g l}(1)) \mathcal{D}(\mathbf{X})^{\mathrm{GL}(1)}=\Omega \mathcal{D}(\mathbf{X})^{\mathrm{GL}(1)}$.

Proof. We wish to apply Lemma 2.11 We give $\mathcal{D}(\mathbf{X})^{\mathrm{GL}(1)}$ the filtration induced from that of $\mathcal{D}(\mathbf{X})$, so hypothesis (i) follows from Lemma 2.10 and [11] while hypothesis (iii) follows from Lemma 2.9 for $K=P$. Thus, only (ii) needs to be checked.

Let $\varpi: \mathbf{X} \rightarrow \mathbf{X} / / \mathrm{GL}(1)$ be the quotient map and pick $f=u_{i} y_{j} \in \mathcal{O}(\mathbf{X})^{\mathrm{GL}(1)}$. Then, $\mathbf{X}_{f}$ is an affine, open subvariety of $\mathbf{X}$ on which GL(1) clearly acts freely. Also, since $f$ is GL(1)-invariant, $\mathbf{X}_{f} / / \mathrm{GL}(1) \cong(\mathbf{X} / / \mathrm{GL}(1))_{f}$. Thus, by [29, Corollary 4.5], $\rho$ induces an isomorphism

$$
\mathcal{D}\left(\mathbf{X}_{f}\right)^{\mathrm{GL}(1)} /(\Omega) \cong \mathcal{D}\left(\mathbf{X}_{f} / / \mathrm{GL}(1)\right)=\mathcal{D}(\mathbf{X} / / \mathrm{GL}(1))_{f}
$$

Since $P$ is generated by such elements $f$, hypothesis (ii) of Lemma 2.11 follows, as required.

Putting the last few results together, we obtain the main technical result of this section:

Proposition 2.13. Let $A=\operatorname{Im}(\rho) \cong \mathcal{D}(\mathbf{X})^{\mathrm{GL}(1)} /(\Omega)$ and write $P$ for the augmentation ideal of $\mathcal{O}(\mathbf{X})^{\mathrm{GL}(1)}$. Then, $\operatorname{GKdim}(A / A P) \leq \operatorname{GKdim}(A)-2$.

Proof. Let $N=\mathcal{D}(\mathbf{X}) /(\mathcal{D}(\mathbf{X}) P+\mathcal{D}(\mathbf{X}) \Omega)$, as in Lemma2.9, Then, Proposition 2.12 implies that $A / A P \cong N^{\mathrm{GL}(1)}$. Thus, by Lemmas 2.3 and 2.9,

$$
\operatorname{GKdim}(A / A P) \leq \operatorname{GKdim}\left(\mathcal{D}(\mathbf{X})^{\mathrm{GL}(1)}\right)-3=\operatorname{GKdim}(A)-2,
$$

as required.

Standard results now lead to the main theorem. Combined with the fact that $\mathcal{D}(\mathbf{X}) \cong U(\mathfrak{s o}(2 n+2)) / J_{0}$ this proves Theorem 0.1 from the introduction.

Theorem 2.14. Assume that $n \geq 3$. Then, $\mathcal{D}(\overline{\mathbb{O}}) \cong \mathcal{D}(\mathbf{X})^{\mathrm{GL}(1)} /(\Omega)$.

Proof. To begin with, regard $A \subseteq D=\mathcal{D}(\overline{\mathbb{O}})$ as modules over $F$. We assume that $A \neq D$. By Corollary 2.2(i), $D$ is an essential extension of $A$ as an $F$-module, while Proposition 2.13 implies that $\operatorname{GKdim}(\mathcal{D}(\overline{\mathbb{O}}) / A) \leq \operatorname{GKdim}(A)-2$. Thus, Lemma 2.10 and [2. Theorem 1.14] imply that $D$ is finitely generated as a left or right $F$-module and hence as a left or right $A$-module (by Lemma 2.10(ii) the tameness hypothesis for $D / A$ in [2] is equivalent to the assertion that $\operatorname{GKdim}(D / A) \leq$ $\operatorname{GKdim}(A)-2$ ). If $D=\sum_{i=1}^{t} d_{i} A$, then Corollary 2.2(ii) implies that $P^{k} d_{i} \subseteq A$ for some $k$. Hence, $K D \subset A$, for $K=A P^{k} A$. By Proposition 2.13, again, $\operatorname{GKdim}(A / K) \leq \operatorname{GKdim}(A)-2$.

By [14, $\overline{\mathbf{O}}_{\text {min }}$ has rational singularities. Thus, by [4], $\overline{\mathbf{O}}_{\text {min }} / / \mathrm{GL}(1)$ has rational singularities and so, in particular, it is Cohen-Macaulay. Of course, $\mathcal{D}(\mathbf{X})^{\mathrm{GL}(1)}$ has associated graded ring $\mathcal{O}\left(\overline{\mathbf{O}}_{\min } / / \mathrm{GL}(1)\right)$. Thus, $\operatorname{gr} A \cong \operatorname{gr}\left(\mathcal{D}(\mathbf{X})^{\mathrm{GL}(1)}\right) /(\operatorname{gr} \Omega)$ is also Cohen-Macaulay, with pure dimension.

Now give $K$ and $A / K$ the induced filtrations from that of $A$. Then, gr $A / \operatorname{gr} K \cong$ $\operatorname{gr}(A / K)$ and so GKdim(gr $A / \operatorname{gr} K) \leq \operatorname{GKdim}(\operatorname{gr} A)-2$, by the first paragraph. As gr $A$ is Cohen-Macaulay with pure dimension, $\mathrm{j}_{A}(A / K) \geq 2$, by [ 5 , Corollary 2.1.4] and [2, Proposition 3.1]; thus $\operatorname{Ext}_{A}^{1}(A / K, A)=0$. However, as $D$ and $A$ are domains with the same quotient division ring, $\operatorname{Ext}_{A}^{1}(D / A, A) \neq 0$. Since $D / A$ is a homomorphic image of $(A / K)^{m}$, for some $m$, the usual long exact sequence in cohomology implies that $\operatorname{Ext}_{A}^{1}(A / K, A) \neq 0$. This contradiction implies that $D=A$ and completes the proof. 
Remark 2.15. Using the ideas in [22] one can show that $\rho$ is graded surjective, when one filters both $\mathcal{D}(\mathbf{X})$ and $\mathcal{D}(\overline{\mathbb{O}})$ by order of differential operator. Equivalently, given any $\theta \in \mathcal{D}(\overline{\mathbb{O}})$, then $\theta=\rho\left(\theta^{\prime}\right)$ for some $\theta^{\prime} \in \mathcal{D}(\mathbf{X})$ of the same order.

\section{The CASE OF $\mathfrak{s l}(2)$}

We assume that $n=2$ throughout this section, but otherwise keep the notation of the last two sections. In particular, $\mathbb{O}=\mathbb{O}_{\min }(\mathfrak{s l}(2))$ and $\mathbf{X}=\left\{(a, b) \in \mathbb{C}^{2} \times \mathbb{C}^{2}\right.$ : $a \cdot b=0\}$. In this case, the proofs of the last section do not work. Nevertheless, the objects in Theorem 2.14 still make sense and so one can ask whether that isomorphism still holds. As we show in this section, the answer is "No."

The real reason for this is that $\mathfrak{s l}(2)=\mathfrak{s p}(2)$ and, as such, $\mathcal{D}(\overline{\mathbb{O}}) \cong A_{2}(\mathbb{C})^{\mathbb{Z}_{2}}$ contains operators of order 2 and Euler degree -1 . Let us make this more explicit. We may identify $\mathcal{O}(\overline{\mathbb{O}}) \cong \mathbb{C}\left[w_{1}^{2}, w_{1} w_{2}, w_{2}^{2}\right]=\mathbb{C}\left[w_{1}, w_{2}\right]^{\mathbb{Z}_{2}}$, where $\mathbb{Z}_{2}=\langle\sigma\rangle$ acts by $\sigma\left(w_{j}\right)=-w_{j}$. It follows easily that

$$
\mathcal{D}(\overline{\mathbb{O}}) \cong \mathcal{D}\left(\mathbb{C}^{2}\right)^{\mathbb{Z}_{2}}=\mathbb{C}\left\langle w_{i} w_{j}, w_{i} \partial_{w_{j}}, \partial_{w_{i}} \partial_{w_{j}}: 1 \leq i, j \leq 2\right\rangle .
$$

We leave it to the reader to check that, if one rewrites the $\partial_{w_{i}} \partial_{w_{j}}$ in terms of the $z_{i j}$ and their derivations one finds that they have Euler degree -1 but, of course, order 2. It is clear that none of the generators of $\mathcal{D}(\mathbf{X})^{\mathbb{C}^{*}}$ given in (1.6) have these properties, and so one should expect that the $\partial_{w_{i}} \partial_{w_{j}} \notin \operatorname{Im}(\rho)$. Unfortunately, it is hard to make this intuitive proof rigorous, even in such a low dimensional case. So, we will give a more theoretical proof that reduces the question to one of torus actions on affine space.

More formally, rewrite

$$
\begin{aligned}
\mathbf{X} & =\left\{\left(a_{1}, a_{2}, b_{1}, b_{2}\right) \in \mathbb{C}^{2} \times \mathbb{C}^{2}: a_{1} b_{1}+a_{2} b_{2}=0\right\} \\
& \cong \mathbf{X}^{\prime}=\left\{M=\left(\begin{array}{cc}
a_{1} & a_{2} \\
b_{2} & -b_{1}
\end{array}\right): \operatorname{det}(M)=0\right\} .
\end{aligned}
$$

By classical invariant theory, $\mathbf{X}^{\prime}$ is isomorphic to the variety of closed orbits $\mathbf{Y}^{\prime} / / \mathbb{C}^{*}$, where $\mathbf{Y}^{\prime}=\mathbb{C}^{2} \times \mathbb{C}^{2}$ with the natural $\mathbb{C}^{*}$-action $\lambda \cdot(\alpha, \beta)=\left(\lambda^{-1} \alpha, \lambda \beta\right)$. Thus, pulling the original action of $\mathbb{C}^{*}$ on $\mathbf{X}$ to one on $\mathbf{Y}^{\prime}$, we find that $\overline{\mathbb{O}} \cong \mathbf{Y}^{\prime} / /\left(\mathbb{C}^{*} \times \mathbb{C}^{*}\right)$, where the action is defined by

$$
(\lambda, \mu) \cdot\left(\alpha_{1}, \alpha_{2}, \beta_{1}, \beta_{2}\right)=\left(\lambda^{-1} \mu^{-1} \alpha_{1}, \lambda^{-1} \mu \alpha_{2}, \lambda \beta_{1}, \lambda \beta_{2}\right) .
$$

Thus, restriction of differential operators provides a homomorphism

$$
\rho_{1}: \mathcal{D}\left(\mathbf{Y}^{\prime}\right)^{\mathbb{C}^{*} \times \mathbb{C}^{*}} \longrightarrow \mathcal{D}\left(\mathbf{Y}^{\prime} / /\left(\mathbb{C}^{*} \times \mathbb{C}^{*}\right)\right)=\mathcal{D}(\overline{\mathbb{O}}) .
$$

Now, $\rho_{2}: \mathcal{D}\left(\mathbf{Y}^{\prime}\right)^{\mathbb{C}^{*} \times\{1\}} \rightarrow \mathcal{D}\left(\mathbf{Y}^{\prime} / /\left(\mathbb{C}^{*} \times\{1\}\right)\right)=\mathcal{D}(\mathbf{X})$ is surjective by [24, Theorem 0.3 and $(0.7)]$. Thus, passing to invariants under $\{1\} \times \mathbb{C}^{*}$, the map $\rho_{2}^{\prime}$ :

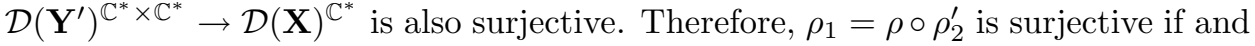
only if $\rho$ is surjective. But, by [26. Theorem B] or [29, Proposition 10.5], $\rho_{1}$ is not surjective.

Hence, we have proved:

Proposition 3.1. When $n=2$, the homomorphism $\mathcal{D}(\mathbf{X})^{\mathrm{GL}(1)} \rightarrow \mathcal{D}(\overline{\mathbb{O}})$ is not surjective. 


\section{LAGRANGIAN SUBMANIFOLDS OF THE MINIMAL ORBIT}

The work of Astashkevich and Brylinski in [1] is a continuation of that of Brylinski and Kostant in [9] where the authors proved analogous results for appropriate Lagrangian submanifolds $\mathbf{L}$ contained in the minimal orbit of a simple Lie algebra, in the sense that [9] proves the existence of an element $D \in \mathcal{D}(\mathbf{L})$ of negative Euler degree. See, also, 6, 7]. There are just two families of these manifolds for classical Lie algebras, one of which is rather trivial. In this section, we show how to modify the results from Section 2 to completely describe $\mathcal{D}(\mathbf{L})$ in these cases.

We begin by describing the varieties. Let $\mathfrak{g}_{\mathbb{R}}$ be a simple, real Lie algebra with a Cartan decomposition $\mathfrak{g}_{\mathbb{R}}=\mathfrak{k}_{\mathbb{R}}+\mathfrak{p}_{\mathbb{R}}$ and complexification $\mathfrak{g}=\mathfrak{k}+\mathfrak{p}$. Following [9], we are interested in the variety $\mathbf{L}=\overline{\mathbb{O}}_{\text {min }}(\mathfrak{g}) \cap \mathfrak{p}$ in the case when the symmetric space corresponding to $\mathfrak{g}_{\mathbb{R}}$ is non-Hermitian and $\mathbf{L} \neq\{0\}$. By the Kostant-Sekiguchi correspondence, this final condition is equivalent to the assumption that $\mathbb{O}_{\min }(\mathfrak{g})$ is the complexification of a nilpotent orbit in $\mathfrak{g}_{\mathbb{R}}$ (see [10 (2.1)]).

By comparing [12, Table $\mathrm{V}$ and $\S \mathrm{X} .6 .3$, p. 518] with [10] Remark 2.2], we find that there are only two cases for $\mathfrak{g}$ classical:

$$
\mathrm{Al}: \quad \mathfrak{g}=\mathfrak{s l}(n) \supset \mathfrak{k}=\mathfrak{s o}(n), \quad n \geq 3
$$

and

$$
\text { BDI : } \quad \mathfrak{g}=\mathfrak{s o}(p+q) \supset \mathfrak{k}=\mathfrak{s o}(p) \times \mathfrak{s o}(q), \quad p \geq q \geq 3 .
$$

The latter is the interesting case; it corresponds to the decomposition

$$
\mathfrak{g}=\left\{\left[\begin{array}{cc}
A & B \\
-B^{t} & C
\end{array}\right]: A=-A^{t} \in \mathrm{M}_{p}(\mathbb{C}) \text { and } C=-C^{t} \in \mathrm{M}_{q}(\mathbb{C})\right\},
$$

with subspaces $\mathfrak{k}=\left\{\left(\begin{array}{cc}A & 0 \\ 0 & C\end{array}\right)\right\}$ and $\mathfrak{p}=\left\{\left(\begin{array}{rr}0 & B \\ -B^{t} & 0\end{array}\right)\right\}$. Since the minimal orbit $\mathbb{O}_{\min }(\mathfrak{g})$ consists of matrices of square zero and rank two, we may identify $\mathbf{L}=\overline{\mathbb{O}}_{\text {min }}(\mathfrak{g}) \cap \mathfrak{p}$ with $p \times q$ matrices of the following form:

$$
\mathbf{L}=\left\{B \in \mathrm{M}_{p, q}(\mathbb{C}): B B^{t}=B^{t} B=0 \text { and } \operatorname{rk}(B) \leq 1\right\} .
$$

Define $\mathbf{W}_{1}=\left\{a \in \mathbb{C}^{p}: a \cdot a=0\right\}$ and $\mathbf{W}_{2}=\left\{b \in \mathbb{C}^{q}: b \cdot b=0\right\}$, with the GL(1) action on $\mathbf{W}=\mathbf{W}_{1} \times \mathbf{W}_{2}$ defined by $\lambda \cdot(a, b)=\left(a \lambda^{-1}, \lambda b\right)$. Just as in Section 1 the multiplication map $\mathbf{W} \rightarrow \mathbf{L}$ given by $(a, b) \mapsto a b^{t}$ induces an isomorphism $\mathbf{L} \cong \mathbf{W} / / \mathrm{GL}(1)$.

Once again, [23] implies that

$$
\mathcal{D}(\mathbf{W}) \cong U(\mathfrak{s o}(p+2)) / J_{0} \otimes U(\mathfrak{s o}(q+2)) / J_{0} .
$$

We now follow the style of argument from Section 2 to determine $\mathcal{D}(\mathbf{L})$. Fix coordinate functions $\left\{x_{i}\right\}$ for $\mathbf{W}_{1}$ and $\left\{y_{i}\right\}$ for $\mathbf{W}_{2}$ so that $\sum_{i} x_{i}^{2}=\sum_{i} y_{i}^{2}=0$. As before, let $P$ denote the augmentation ideal of $\mathcal{O}(\mathbf{L})^{\mathrm{GL}(1)}$, and set $\Omega=\sum_{i}\left(x_{i} \partial_{x_{i}}-y_{i} \partial_{y_{i}}\right)$. Thus, $\Omega \in \operatorname{Ker}(\rho)$, for the natural map

$$
\rho: \mathcal{D}(\mathbf{W})^{\mathrm{GL}(1)} \longrightarrow \mathcal{D}(\mathbf{W} / / \mathrm{GL}(1))=\mathcal{D}(\mathbf{L}) .
$$

Lemma 4.1. Let $I=\sum_{i=1}^{p} \mathcal{D}(\mathbf{W}) x_{i}+\mathcal{D}(\mathbf{W})(\Omega-\lambda)$, for some $\lambda \in \mathbb{C}$. Then, $\operatorname{GKdim}(\mathcal{D}(\mathbf{W}) / I) \leq \operatorname{GKdim}(\mathcal{D}(\mathbf{W}))-3$.

Proof. Let $I^{\prime}=\sum_{i=1}^{p} \mathcal{D}\left(\mathbf{W}_{1}\right) x_{i}$ and give each $\mathcal{D}\left(\mathbf{W}_{i}\right)$ the filtration induced from that of $U(\mathfrak{s o}(r+2))$, for $r=p$ or $r=q$. Thus

$$
\operatorname{gr}(\mathcal{D}(\mathbf{W}) / I) \cong\left(\operatorname{gr} \mathcal{D}\left(\mathbf{W}_{1}\right) / \operatorname{gr} I^{\prime} \otimes \operatorname{gr} \mathcal{D}\left(\mathbf{W}_{2}\right)\right) /(\operatorname{gr} \Omega) .
$$


Now, 23] Theorem 4.7] implies that $\operatorname{GKdim}\left(\mathcal{D}\left(\mathbf{W}_{1} / I^{\prime}\right)\right) \leq \operatorname{GKdim}\left(\mathcal{D}\left(\mathbf{W}_{1}\right)\right)-2$; and so $A=\operatorname{gr} \mathcal{D}\left(\mathbf{W}_{1}\right) / \operatorname{gr} I^{\prime} \otimes \operatorname{gr} \mathcal{D}\left(\mathbf{W}_{2}\right)$ satisfies $\operatorname{GKdim}(A) \leq \operatorname{GKdim}(\mathcal{D}(\mathbf{W}))-2$. On the other hand, by [23], again, gr $\mathcal{D}\left(\mathbf{W}_{2}\right) \cong \mathcal{O}\left(\overline{\mathbb{O}}_{\min }(\mathfrak{s o}(q+2))\right)$ and this ring is a domain, by [11. However, gr $\Omega=\Omega_{1}+\Omega_{2}$, where $\Omega_{1} \in \operatorname{gr} \mathcal{D}\left(\mathbf{W}_{1}\right) / \operatorname{gr} I^{\prime}$ and $0 \neq \Omega_{2} \in \operatorname{gr} \mathcal{D}\left(\mathbf{W}_{2}\right)$. It follows immediately that $\operatorname{GKdim}(A /(\operatorname{gr} \Omega))<\operatorname{GKdim}(A)$, as required.

Lemma 4.2. Let $M=\mathcal{D}(\mathbf{W})^{\mathrm{GL}(1)} \Omega+\mathcal{D}(\mathbf{W})^{\mathrm{GL}(1)} P$. Then,

$$
\operatorname{GKdim}\left(\mathcal{D}(\mathbf{W})^{\mathrm{GL}(1)} / M\right) \leq \operatorname{GKdim}\left(\mathcal{D}(\mathbf{W})^{\mathrm{GL}(1)}\right)-3 .
$$

Proof. We use the proof of Lemma 2.9 Set $N=\mathcal{D}(\mathbf{W}) /(\mathcal{D}(\mathbf{W}) \Omega+\mathcal{D}(\mathbf{W}) P)$. As in Lemma 2.9, using Lemma 4.1 one sees that $\operatorname{GKdim}(N) \leq \operatorname{GKdim}(\mathcal{D}(\mathbf{W}))-3$. If $\operatorname{GKdim}\left(N^{\mathrm{GL}(1)}\right)=\operatorname{GKdim}(N)-1$, the result follows from $\operatorname{GKdim}\left(\mathcal{D}(\mathbf{W})^{\mathrm{GL}(1)}\right)=$ $\operatorname{GKdim}(\mathcal{D}(\mathbf{W}))-1$. Suppose that $\operatorname{GKdim}\left(N^{\mathrm{GL}(1)}\right)=\operatorname{GKdim}(N)$ and set $I=$ $\operatorname{gr}(\mathcal{D}(\mathbf{W}) \Omega+\mathcal{D}(\mathbf{W}) P)$. Then, as in Lemma 2.8, one finds that

$$
\mathcal{V}(I) \subset\left[\overline{\mathbb{O}}_{\min }(\mathfrak{s o}(p+2)) \times \overline{\mathbb{O}}_{\min }(\mathfrak{s o}(q+2))\right]^{\mathrm{GL}(1)} .
$$

The definition of the GL(1)-action and the identification of $\mathfrak{s o}(p+2)$ inside $\mathcal{D}\left(\mathbf{W}_{1}\right)$ given in [21, 1.2.1] imply that

$$
[\mathfrak{s o}(p+2) \times \mathfrak{s o}(q+2)]^{\mathrm{GL}(1)} \equiv\left(\mathfrak{s o}(p) \oplus \mathbb{C} I_{p}\right) \times\left(\mathfrak{s o}(q) \oplus \mathbb{C} I_{q}\right)
$$

where $I_{p}, I_{q}$ are semisimple elements of $\mathfrak{s o}(p+2)$, respectively $\mathfrak{s o}(q+2)$. It follows that if $(a, b) \in \mathcal{V}(I)$, then $a$ identifies with a nilpotent element of square zero and rank at most two in $\mathfrak{s o}(p)$ (similarly with $b$ in $\mathfrak{s o}(q)$ ). Recall that, for $r \geq 5$, the dimension of $\mathbb{O}_{\min }(\mathfrak{s o}(r))$ is $2 r-6$. Hence, if $p \geq q \geq 5$ we have

$$
\operatorname{dim} \mathcal{V}(I) \leq(2 p-6)+(2 q-6) \leq 2(p+q)-8=\operatorname{GKdim}\left(\mathcal{D}(\mathbf{W})^{\mathrm{GL}(1)}\right)-3 .
$$

We leave to the reader the easy verification that $\operatorname{dim} \mathcal{V}(I) \leq \operatorname{GKdim}\left(\mathcal{D}(\mathbf{W})^{\mathrm{GL}(1)}\right)-3$ also holds when $q \leq 4$. (Use the identities $\mathfrak{s o}(3) \cong \mathfrak{s l}(2)$ and $\mathfrak{s o}(4) \cong \mathfrak{s l}(2) \times \mathfrak{s l}(2)$ combined with the fact that $\operatorname{dim} \mathbb{O}_{\min }(\mathfrak{s l}(2))=2$.)

Proposition 4.3. $\operatorname{Ker}(\rho)=\tau(\mathfrak{g l}(1)) \mathcal{D}(\mathbf{W})^{\mathrm{GL}(1)}=\Omega \mathcal{D}(\mathbf{W})^{\mathrm{GL}(1)}$.

Proof. As in the proof of Proposition 2.12, we use Lemma 2.11 and we only need to check hypothesis (ii) of that result (clearly the statement and proof of Lemma 2.10 also hold for $\left.\mathcal{D}(\mathbf{W})^{\mathrm{GL}(1)}\right)$.

Let $\varpi: \mathbf{W} \rightarrow \mathbf{W} / / \mathrm{GL}(1)$ denote the quotient map and pick $f=x_{i} y_{j} \in$ $\mathcal{O}(\mathbf{W})^{\mathrm{GL}(1)}$. Just as in the proof of Proposition 2.12, $\mathbf{W}_{f}$ is an affine $\varpi$-saturated open subset of $\mathbf{W}$ on which GL(1) acts freely. Hypothesis (ii) then follows from [29, Corollary 4.5] and the fact that $P$ is generated by such elements $f$.

Theorem 4.4. Assume that $(\mathfrak{g}, \mathfrak{k})=(\mathfrak{s o}(p+q), \mathfrak{s o}(p) \times \mathfrak{s o}(q))$, for $p \geq q \geq 3$. Then, in the notation defined above, $\mathcal{D}(\mathbf{L}) \cong \mathcal{D}(\mathbf{W})^{\mathrm{GL}(1)} /(\Omega)$.

Proof. First note that, using the proof of Proposition 2.13, $\operatorname{GKdim}(A / A P) \leq$ $\operatorname{GKdim}(A)-2$, for $A=\operatorname{Im}(\rho)$. The proof of Theorem 2.14 now goes through mutatis mutandis.

For the record we note the differential operators of negative Euler degree in $\mathcal{D}(\mathbf{L})$ that this theorem produces. Since we have used a different quadratic form than 
that used in Section 2, we first need to write down some operators from $\mathcal{D}(\mathbf{W})$. Let $\Delta^{\prime}=\frac{1}{2} \sum_{i=1}^{p} \partial_{x_{i}}^{2}$ and $E^{\prime}=\sum_{i=1}^{p} x_{i} \partial_{x_{i}} \in \mathcal{D}\left(\mathbf{W}_{1}\right)$ and define

$$
\Theta_{j}^{x}=x_{j} \Delta^{\prime}-\partial_{x_{j}}\left(E^{\prime}+(p-4) / 2\right) .
$$

Then, [21, Lemme 1.1.4] implies that $\Theta_{j}^{x} \in \mathcal{D}\left(\mathbf{W}_{1}\right)$. Let $\Theta_{j}^{y}$ denote the analogous element from $\mathcal{D}\left(\mathbf{W}_{2}\right)$, obtained by replacing $x$ by $y$ and $p$ by $q$. Clearly,

$$
\Theta_{i}^{x} \Theta_{j}^{y} \in \mathcal{D}(\mathbf{W})^{\mathrm{GL}(1)} \quad \text { for } 1 \leq i \leq p \text { and } 1 \leq j \leq q .
$$

The analysis from the end of Section 1 extends to show that the $\rho\left(\Theta_{i}^{x} \Theta_{j}^{y}\right)$ are elements of $\mathcal{D}(\mathbf{L})$ of Euler degree -1 and order 4, that they commute and that they span a subspace of $\mathcal{D}(\mathbf{L})$ isomorphic to $\mathfrak{p}$ as $\mathfrak{k}$-module. This proves the heart of [9, Theorem 3.10] for this case. We leave it to the reader to extend the arguments from Section 1 to find a complete set of generators of $\mathcal{D}(\mathbf{L})$.

We end the section by considering the Lagrangian variety $\mathbf{L}=\overline{\mathbb{O}}_{\text {min }}(\mathfrak{g}) \cap \mathfrak{p}$ for the case $\mathrm{Al}: \mathfrak{g}=\mathfrak{s l}(n) \supset \mathfrak{k}=\mathfrak{s o}(n)$. Here, $\mathfrak{p}$ is the set of symmetric $n \times n$ matrices and so $\mathbf{L}$ becomes the set of $n \times n$ symmetric matrices of rank at most one and square zero. As such, we may identify $\mathbf{L}=\mathbf{X} / / \mathrm{O}(1)$, for $\mathbf{X}=\left\{a \in \mathbb{C}^{n}: a \cdot a=0\right\}$, via the map $a \mapsto a a^{t}$ from $\mathbf{X}$ to $\mathbf{L}$.

Proposition 4.5. Assume that $(\mathfrak{g}, \mathfrak{k})=(\mathfrak{s l}(n), \mathfrak{s o}(n))$, for $n \geq 3$. Then, in the above notation, restriction of functions yields an isomorphism

$$
\mathcal{D}(\mathbf{X})^{\mathrm{O}(1)} \stackrel{\sim}{\sim} \mathcal{D}(\mathbf{L}) .
$$

Proof. Since $\mathrm{O}(1)=\langle\sigma\rangle$ acts on $\mathbf{X}$ by $\sigma(x)=-x$, the fixed point set in $\mathbf{X}$ is $\{0\}$, which has codimension at least two in $\mathbf{X}$. The result now follows from 13 Corollary 1.3].

\section{A NON-Minimal NilPotent ORBit}

For the final result of this paper, we show how the earlier results can also be used to describe the ring of differential operators over a non-minimal nilpotent orbit in $\mathfrak{s p}(2 n)$. In fact, this is closely related to the earlier work, since the orbit we consider is a "shared orbit" with the minimal orbit of $\mathfrak{s l}(2 n)$, in the sense of [8]. See, in particular, 8, Theorem 5.9(iii)].

Set $J_{n}=\left(\begin{array}{cc}0 & I_{n} \\ -I_{n} & 0\end{array}\right) \in \mathrm{M}_{2 n}(\mathbb{C})$ and let $\vartheta$ be the involutive automorphism of $\mathfrak{s l}(2 n)$ defined by $\vartheta(X)=J_{n} X^{t} J_{n}$. Recall that $\mathfrak{s p}(2 n)=\{X \in \mathfrak{s l}(2 n): \vartheta(X)=X\}$, thus $\eta=I+\vartheta$ gives a surjective linear map from $\mathfrak{s l}(2 n)$ onto $\mathfrak{s p}(2 n)$. Set

$$
\mathbb{O}_{2}=\left\{X \in \mathfrak{s p}(2 n): X^{2}=0 \text { and } \operatorname{rk} X=2\right\} .
$$

Hence, $\overline{\mathbb{O}}_{2} \backslash \mathbb{O}_{2}=\overline{\mathbb{O}}_{\text {min }}(\mathfrak{s p}(2 n))$ and, in fact, $\mathbb{O}_{2}$ is the next to minimal orbit in the sense that it is contained in all other nilpotent orbit closures. Write $\mathbb{O}=$ $\mathbb{O}_{\min }(\mathfrak{s l}(2 n))$ for the minimal nilpotent orbit in $\mathfrak{s l}(2 n)$, and note that $\mathbb{O}$ is $\vartheta$-stable. The following lemma is not difficult to prove.

Lemma 5.1. The map $\eta$ induces an isomorphism: $\overline{\mathbb{O}} / /\langle\vartheta\rangle \stackrel{\sim}{\longrightarrow} \overline{\mathbb{O}}_{2}$.

Recall that $\mathcal{D}(\overline{\mathbb{O}})$ has been described in Theorem 0.1 . From this result one can deduce a complete description of $\mathcal{D}\left(\overline{\mathbb{O}}_{2}\right)$ :

Proposition 5.2. Restriction of functions yields an isomorphism

$$
\mathcal{D}(\overline{\mathbb{O}})^{\langle\vartheta\rangle} \stackrel{\sim}{\sim} \mathcal{D}\left(\overline{\mathbb{O}}_{2}\right) \text {. }
$$


Proof. Observe that $\overline{\mathbb{O}}_{1}=\{X \in \overline{\mathbb{O}}: \vartheta(X)=X\}$ is the closure of the minimal nilpotent orbit in $\mathfrak{s p}(2 n)$, which has dimension $2 n$. Thus the codimension of $\overline{\mathbb{O}}_{1}$ in $\overline{\mathbb{O}}$ is $2 n-2 \geq 2$. The result then follows from Lemma 5.1] and [13, Corollary 1.3].

\section{Questions}

The most obvious questions are:

Question 6.1. Does there exist an analogue to Theorem 0.1 for the ring of differential operators $\mathcal{D}\left(\mathbb{O}_{\min }(\mathfrak{s o}(n))\right)$ ?

Question 6.2. In the notation of Section 4 does there exist an analogue to Theorem 4.4 for the ring of differential operators $\mathcal{D}(\mathbf{L})$ over the exceptional Lie algebras? Ideally, there should exist a uniform approach for describing these rings.

The natural approach to Question 6.1 is to again regard $\overline{\mathbb{O}}_{\min }(\mathfrak{s o}(n))$ as a categorical quotient. By [17 Theorem 3.3] and [18. Theorem 5.3], any nilpotent orbit in a classical simple Lie algebra has such a description and in this case it is easy to describe: Set

$$
\mathbf{V}=\left\{A=\left[\begin{array}{l}
a \\
b
\end{array}\right] \in \mathrm{M}_{2, n}(\mathbb{C}): a \cdot a=b \cdot b=a \cdot b=0\right\},
$$

and let $\operatorname{Sp}(2)$ act on $\mathbf{V}$ by left multiplication. Then the map $A \mapsto A^{t} J_{1} A$ yields an isomorphism $\mathbf{V} / / \operatorname{Sp}(2) \stackrel{\sim}{\sim} \overline{\mathbb{O}}_{\min }(\mathfrak{s o}(n))$. Thus a more precise formulation of 6.1 is:

Question 6.3. (i) Is $\mathcal{D}\left(\mathbb{O}_{\min }(\mathfrak{s o}(n))\right) \cong\left(\mathcal{D}(\mathbf{V}) / \mathcal{D}(\mathbf{V}) \tau(\mathfrak{s p}(2))^{\mathrm{Sp}(2)}\right.$ ?

(ii) Describe $\mathcal{D}(\mathbf{V})$.

Unfortunately we are unable to solve either part of 6.3 Thus, even though $\mathbf{V}$ has a simple description, we can say very little about $\mathcal{D}(\mathbf{V})$. In contrast to the situation for $\mathcal{D}(\mathbf{X})$ in $\ 2$, we know of no way to (for example) identify $\mathcal{D}(\mathbf{V})$ with $U(\mathfrak{g}) / J$ for some Lie algebra $\mathfrak{g}$, and ideal $J$.

As mentioned in the introduction, Astashkevich and Brylinski [1] also find a differential operator $D_{0}$ of order 4 and Euler degree -1 inside $\mathcal{D}\left(\mathbb{O}_{\min }(\mathfrak{s o}(n))\right)$. If 6.3(i) is true, one can hope that the preimage of $D_{0}$ inside $\mathcal{D}(\mathbf{V})$ has a pleasant form. Here, again, the situation is more complicated than that for $\mathfrak{s t}(n)$. Indeed, recall from Proposition 1.2 that, for $\mathfrak{s l}(n), D_{0}=\rho\left(-\frac{1}{4} \Phi_{n} \Theta_{1}\right)$ where the $\Phi_{i}$ and $\Theta_{j}$ had order 2 and degree -1 . In contrast, a straightforward but tedious computation, which we omit, proves:

Lemma 6.4. There is no element in $\mathcal{D}(\mathbf{V})$ of order 2 and Euler degree -1.

One can also ask whether other nilpotent orbits have pleasant rings of differential operators. Specifically we ask:

Question 6.5. Let $\mathfrak{g}$ be a semisimple Lie algebra.

(i) For which nilpotent orbits $\mathbf{O}$ is $\mathcal{D}(\overline{\mathbf{O}})$ Noetherian or simple?

(ii) Describe $\mathcal{D}(\mathbf{N})$ for the nilcone $\mathbf{N}$.

Notice that if $\mathcal{D}(\overline{\mathbf{O}})$ is simple, then $\overline{\mathbf{O}}$ has to be Cohen-Macaulay 31, Theorem 6.2.5] and therefore normal [18, 10.1], so we are mostly concerned with normal orbits in [6.5. We remark that it follows trivially from Theorem 0.1 that $\mathcal{D}(\overline{\mathbb{O}})$ is Noetherian, and it is easy to show that $\mathcal{O}\left(\overline{\mathbb{O}}_{\text {min }}(\mathfrak{s l}(n))\right)$ is a simple $\mathcal{D}\left(\overline{\mathbb{O}}_{\text {min }}(\mathfrak{s l}(n))\right)$ module, but we cannot answer:

Question 6.6. Is the ring $\mathcal{D}\left(\overline{\mathbb{O}}_{\min }(\mathfrak{s l}(n))\right)$ of Theorem 0.1 simple? 


\section{ACKNOWLEDGEMENT}

This research was conducted while the first author was visiting the University of Michigan and was partially supported by the NSF, and also while the second author was visiting and partially supported by the University of Brest. They would like to thank all three institutions.

\section{REFERENCES}

1. A. Astashkevich and R. Brylinski, Exotic Differential Operators on Complex Minimal Nilpotent Orbits, in "Advances in Geometry", (Progress in Math., Vol. 172), Birkhäuser, Boston, 1998. CMP 99:07

2. J.-E. Björk, The Auslander condition on Noetherian rings, in "Séminaire d'Algèbre P. Dubreil et M.-P. Malliavin" (Lecture Notes in Math. No. 1404), Springer-Verlag, Berlin/New York, 1989. MR 90m:16002

3. W. Borho and H. Kraft, Über die Gelfand-Kirillov Dimension, Math. Annalen, 220 (1976), 1-24. MR 54:367

4. J.-F. Boutot, Singularités rationnelles et quotients par les groupes réductifs, Inventiones Math., 88 (1987), 65-68. MR 88a:14005

5. W. Bruns and J. Herzog, Cohen-Macaulay rings, Cambridge Univ. Press, Cambridge, 1996. MR 95h:13020

6. R. Brylinski and B. Kostant, Minimal representations of $E_{6}, E_{7}$ and $E_{8}$ and the generalized Capelli identity, Proc. Nat. Acad. Sci. USA, 91 (1994), 2469-2472. MR 96a:22026

7. Minimal representations, geometric quantization and unitarity, Proc. Nat. Acad. Sci. USA, 91 (1994), 6026-6029. MR 95d:58059

8. (1994), 269-298. MR 94g:22031

9. L Differential Operators on conical Lagrangian manifolds, in "Lie Theory and Geometry: in Honor of B. Kostant", (Progress in Math., Vol. 123), Birkhäuser, Boston, 1994. MR 96h:58076

10. Lagrangian models of minimal representations of $E_{6}, E_{7}$ and $E_{8}$, in "Functional Analysis on the Eve of the 21st Century: in Honor of I. M. Gelfand", (Progress in Math., Vol. 131), Birkhäuser, Boston, 1995. MR 96m:22025

11. D. Garfinkle, A new construction of the Joseph ideal, Ph. D. Thesis, M.I.T., 1982.

12. S. Helgason, Differential Geometry, Lie Groups and Symmetric Spaces, Academic Press, New York, 1978. MR 80k:53081

13. Y. Ishibashi, Nakai's conjecture for invariant subrings, Hiroshima Math. J., 15 (1985), 429436. MR 87b:13003

14. G. Kempf, On the collapsing of homogeneous bundles, Inventiones Math., 37 (1976), 229-239. MR 54:12799

15. G. R. Krause and T. H. Lenagan, Growth of Algebras and Gelfand-Kirillov Dimension, Pitman, Boston 1985. MR 86g:16001

16. H. Kraft, Geometrische Methoden in der Invarianttentheorie, Vieweg, 1984. MR 86j:14006

17. H. Kraft and C. Procesi, Closures of conjugacy classes of matrices are normal, Inventiones Math., 53 (1979), 227-247. MR 80m:1403

18. (1982), 539-602. MR 85b:14065

19. T. Levasseur, Anneaux d'opérateurs differentiels in "Séminaire d'Algèbre P. Dubreil et M.P. Malliavin" (Lecture Notes in Math. No. 867), Springer-Verlag, Berlin/New York, 1981. MR 84j:32009

20. 1519-1532. MR 83k:13006

21. _ La dimension de Krull de $U(s l(3))$, J. Algebra, 102 (1986), 39-59. MR 87m:17019

22. _ Relèvements d'opérateurs différentiels sur les anneaux d'invariants in "Colloque en l'honneur de J. Dixmier" (Progress in Math., Vol. 92), Birkhäuser, Boston, 1990. MR 92f: 16033

23. T. Levasseur, S. P. Smith and J. T. Stafford, The minimal nilpotent orbit, the Joseph ideal and differential operators, J. Algebra, 116 (1988), 480-501. MR 89k:17028 
24. T. Levasseur and J. T. Stafford, Rings of Differential Operators on Classical Rings of Invariants, Mem. Amer. Math. Soc. 81, No. 412, 1989. MR 90i:17018

25. M. Lorenz, Gelfand-Kirillov Dimension, Cuadernos de Algebra, No. 7 (Grenada, Spain), 1988.

26. I. M. Musson, Rings of differential operators on invariant rings of tori, Trans. Amer. Math. Soc., 303 (1987), 805-827. MR 88m:32019

27. D. I. Panyushev, Rationality of singularities and the Gorenstein property for nilpotent orbits, Funct. Anal. Appl., 25 (1991), 225-226. MR 92i:14047

28. V. L. Popov and E. B. Vinberg, Invariant Theory, in "Algebraic Geometry IV", (Eds: A. N. Parshin and I. R. Shafarevich), Springer-Verlag, Berlin/Heidelberg/New York, 1991.

29. G. W. Schwarz, Lifting differential operators from orbit spaces, Ann. Sci. École Norm. Sup., 28 (1995), 253-306. MR 96f:14061

30. R. P. Stanley, Hilbert functions of graded algebras, Adv. in Math., 28 (1978), 57-83. MR 58:5637

31. M. Van den Bergh, Differential operators on semi-invariants for tori and weighted projective spaces, in "Séminaire d'Algèbre P. Dubreil et M.-P. Malliavin" (Lecture Notes in Math. No. 1478), Springer-Verlag, Berlin/New York, 1991. MR 93h:16046

Département de Mathématiques, Université de Brest, 29285 Brest, France

E-mail address: Thierry.Levasseur@univ-brest.fr

Department of Mathematics, University of Michigan, Ann Arbor, Michigan 48109

E-mail address: jts@math.Isa.umich.edu 